\title{
Radius distraction could contribute to long-term stability for treating distal radioulnar joint instability during volar plating of distal radius fracture: a retrospective case series study
}

Cheng-Yu Yin

Taipei Veterans General Hospital

Hui-Kuang Huang

Chia-Yi Christian Hospital

Duretti Fufa

Hospital for Special Surgery

Jung-Pan Wang ( $\sim$ jpwang801@gmail.com )

Taipei Veterans General Hospital

\section{Research Article}

Keywords: distal radioulnar joint instability, distal radius fracture, ulnar styloid fracture, distraction

Posted Date: June 29th, 2021

DOl: https://doi.org/10.21203/rs.3.rs-617556/v1

License: (c) (1) This work is licensed under a Creative Commons Attribution 4.0 International License.

Read Full License 


\section{Abstract}

\section{Background}

The surgical technique of radius distraction for stabilization of distal radioulnar joint (DRUJ) if intraoperative DRUJ instability was found after the fixation of distal radius fracture has been previously described, but this surgical technique lacks clinical and radiographic effect in minimal 3 years follow-up. We therefore evaluated the clinical outcome and radiographic results of radius distraction in minimal 3 years follow-up.

Methods

We reviewed the case series of distal radius fracture with concomitant DRUJ instability receiving radius distraction from the senior author over a 5-year period (January 1st, 2013 to June 30th, 2017) retrospectively, and the evaluation of clinical and radiographic outcomes was performed at clinic as longterm follow-up; a total 34 patients had been evaluated.

Results

At minimal post-operative 36 months follow-up, all cases demonstrated acceptable wrist range of motion with stable DRUJs and low NRS of wrist pain (0.6, SD 0.7) and DASH score (mean 9.1, SD 6.2), and there were no cases suffering from nonunion of distal radius. The mean ulnar variance of injured wrist and uninjured wrist were $-1.2 \mathrm{~mm}$ and $0.2 \mathrm{~mm}$, respectively (SD 1.0 and 0.6 ) with significant statistical difference.

\section{Conclusions}

Radius distraction during volar fixation of distal radius fracture should be consider if DRUJ instability was found by the radioulnar stress test intraoperatively, and the long-term DRUJ stability could be achieved by maintenance of normal-to-negative ulnar variance, with decreased wrist pain and satisfactory function outcome.

Level of Evidence

Therapeutic Level IV

\section{Introduction}

The soft-tissue stabilizers of distal radioulnar joint (DRUJ) include the triangular fibrocartilaginous complex, the radioulnar ligaments, ulnocarpal ligaments, extensor carpi ulnaris subsheath, pronator quadratus, and the interosseous membrane. Among them, the triangular fibrocartilaginous complex (TFCC) is the main intrinsic stabilizer, with its radioulnar ligament originating from the sigmoid notch, across DRUJ, and inserted into the base of ulnar styloid and the fovea (1). Based on the anatomy of the 
TFCC, distal radius fracture (DRF) with concomitant TFCC tear or fracture of ulna styloid could lead to significant DRUJ instability (2-4), and TFCC tears with or without DRUJ instability is also the common pathoanatomy of chronic wrist pain, causing palpable click during the rotation of forearm (5). Although not all studies have found difference in clinical outcome, DRUJ instability has been identified as a poor prognostic factor in some outcomes after DRFs (6).

Although the TFCC is considered as the primary stabilizer for DRUJ (1), it is possible that the DRUJ stability could be maintained by intact extrinsic soft-tissue stabilizers in the setting of complete TFCC tear (7). Moreover, the biomechanical study by Arimitsu et al had suggested that the DRUJ could be stabilized by increased tension of the distal interosseous membrane (DIOM) from ulnar shortening, especially in the presence of distal oblique bundle (DOB) (8). Based on this concept, the surgical technique of radius distraction to treat DRF with concomitant DRUJ instability by DOB tightening was developed (9). Although encouraging early results have been mentioned, no clinical outcomes beyond at least 3 years have been published, and the longevity of the stabilizing effect of tightened DOB was also unknown. Therefore, the aim of this study is to report the outcomes of radius distraction with a minimal post-operative 36 months follow-up.

\section{Materials And Methods}

We retrospectively reviewed the outcomes in a cohort of the patients of DRF with concomitant DRUJ instability necessitating radius distraction between January 1st, 2013 and June 30th, 2017. This study has been performed in accordance with the ethical standards in the 1964 Declaration of Helsinki and has been carried out in accordance with relevant regulations of the US Health Insurance Portability and Accountability Act (HIPAA). The study was approved as simple retrospective study by the Institutional Review Board of the senior author's hospital.

Among the patients with DRFs necessitating surgical intervention, the indications of radius distraction were: (1) presence of concomitant ulnar styloid fracture; (2) subluxation of DRUJ with more than $50 \%$ of sagittal translation ratio from lateral view of radiograph (10); and (3) intraoperative DRUJ instability with more than or equal to Grade 2 of DRUJ instability confirmed by radioulnar stress test (11) compared with the contralateral wrist. Contraindication of radius distraction included: (1) AO-OTA type 2R3B type fracture as the distraction would cause articular step-off; (2) history of previous wrist trauma or arthritis; (3) open fracture or additional intercarpal ligament injury in the same limb. We also excluded intraoperative DRUJ instability that couldn't be corrected by radius distraction alone, bilateral DRFs, and soft tissue injury of the contralateral wrist causing DRUJ instability. Between the retrospective study period, there were in total 76 patients suffered from DRF necessitating surgical intervention, and 39 of 76 patients met the indications of radius distraction. After we excluded three of 39 patients who could not be treated by radius distraction alone and two of 39 patients who were lost to follow-up at senior author's clinic visit, the final cohort was 34 patients with 34 surgical procedures of radius distraction.

\section{Surgical technique}


We performed radius distraction modified from the technique previously described (9). We made open reduction and internal fixation for DRF under general anesthesia with pneumatic tourniquet application in the supine position. Via the flexor carpi radialis approach to the distal radius, we applied the volar locking distal radius plate (Variable Angle LCP Two-Column Volar Distal Radius Plate 2.4., Depuy Synthes, Oberdorf, Switzerland) to the volar side of metaphyseal area of distal radius after the pronator quadratus flap was elevated from the radial boarder. After the metaphyseal fragments of DRF were reduced anatomically and all the distal row of locking screw holes were fixed with locking screws, we then fastened one compression screw in the middle or distal side of oblong hole for provisional anatomic reduction to preserve the length for radius distraction. Then the radioulnar stress test was performed (12) with the patient's elbow in 90-degree flexion and forearm in neutral position. If the radioulnar stress test revealed DRUJ instability or subluxation, we then loosened the compression screw in the oblong hole, moving the locking plate distally till the compression screw hole met the proximal side of oblong hole, and we then fastened the compression screw again. Figure 1 was intraoperative demonstration of radius distraction. We judged the final distance of radius distraction from the end point when the radioulnar stress test met Grade 0 of DRUJ instability (stable DRUJ with moderate resistance) and we may compare the ulnar variance of the injured wrist with that of the contralateral uninjured wrist from intraoperative fluoroscopy in the same neutral posture if positive value of ulnar variance of the injured wrist was still noted after radius distraction to ensure whether this is caused by the innate ulnar variance. Supplement video 1 was the demonstration of radioulnar stress test before and after radius distraction. Finally, we checked ROM of the injured wrist to ensure full pronation and supination after the surgery.

\section{Postoperative management}

All patients were immobilized in a below-elbow volar short arm thermoplastic splint after the surgery. We taught the patients to do active range of motion exercise of elbow and digits on postoperative day one. We encouraged the patients to do light daily activities while wearing the splint. The patients could discard the splint in 1 month postoperatively. Return to full strength of sport and work were allowed at nearly 12 weeks when union of distal radius fracture was confirmed as defined by trabecular bridging across the fracture site on plain radiographs as previous literature described (13).

\section{Evaluations}

We recorded and summarized the demographic data, including patient number, duration from injury to surgery, age, gender, hand side of injured wrist, number of dominant hands, fracture classification, number of ulnar styloid fracture, total operative time, and follow-up period, in Table 1. For follow-up arrangement, patients were seen at two weeks, one, two to six months, and then annually; the final callback evaluation including radiographic, patient-rated and functional outcomes was performed at the final follow-up, which was with minimal post-operative 36 months follow-up. 
Table 1

Patient demographic characteristics

\begin{tabular}{|ll|}
\hline Patient volume, number & 34 \\
\hline Mean Duration from injury to surgery, days (range; SD) & 5 (0 to 11; 3) \\
\hline Mean Age, years (range; SD) & $55(25$ to $75 ; 15)$ \\
\hline Gender, number (male to female) & $10 / 24$ \\
\hline Hand side, number (right to left) & $23 / 11$ \\
\hline Dominant hand, number (percentage of all cases) & $25(73.5 \%)$ \\
\hline Fracture AO type, number & 9 \\
\hline 2R3A2 & 8 \\
\hline 2R3A3 & 7 \\
\hline 2R3C1 & 10 \\
\hline 2R3C2 & \\
\hline Ulnar styloid fracture, number (percentage of all cases) & 24 (70.6\%) \\
\hline Fracture gap after radius distraction, mm (range, SD) & $2.2(1.7$ to $3.1 ; 0.3)$ \\
\hline Total operative time, minutes (range; SD) & 54 (48 to 65; 5) \\
\hline Follow-up period, months (range; SD) & 44 (37 to 56; 5) \\
\hline SD: standard deviation & \\
\hline
\end{tabular}

For radiographic outcome measures, we routinely checked ulnar variance and bone union of the injured wrist on posteroanterior view and lateral view of radiographs in the same neutral posture, and the ulnar variance of the contralateral wrist was also checked at the final clinic visit. These radiographs were taken at each clinic visit after the operation and the radiographic evaluations were done by the senior author.

For patient rated outcome, pain was measured by the numeric rating scale (NRS) of pain, which is an 11point numeric scale from 0 representing "no pain" to 10 representing "worst pain imaginable." We used the Disabilities of Arm, Shoulder and Hand (DASH) questionnaires to evaluate general disabilities of upper extremity, scoring from 0 to 100 , where higher scores indicate more disability of upper extremity. We also used Mayo modified wrist score (MMWS) to evaluate the disability of wrist, where lower scores indicate more disability of the wrist.

For functional outcome measures at the final recalled evaluation, the DRUJ instability was graded by radioulnar stress test as Grade 0 for stable DRUJ, Grade 1 for DRUJ limited instability (increased translation with firm end point), Grade 2 for dynamic DRUJ instability (increased translation without firm end point), and Grade 3 for spontaneous subluxation during active wrist rotation (12). The test for 
detecting late DRUJ instability was performed by the senior author and the first/second authors as blinded evaluators to conclude the exact grading; repeated assessments by three authors might be arranged if no initial conclusion of grading was determined from three authors. The ROM of the wrist (extension, flexion, radial and ulnar deviation, supination, and pronation) was measured with the standard goniometer by the senior author.

\section{Statistical analysis}

All the continuous variables of radiographic measurements and functional outcomes were reported using means with standard deviations. The Spearman's rank-order correlation coefficient was used to evaluate whether there was a statistical correlation between fracture gap after radius distraction and union time. The Wilcoxon signed rank test was used to compare (1) the difference of ulnar variance of injured wrist between the post-operative one-month follow-up and the final follow-up (2) the difference of ulnar variance at the final follow-up between injured and uninjured wrist. A level of significance was set at a $p$ value less than 0.05 .

\section{Results}

In our cohort of 34 cases, mean age of patients at the time of surgery was 55 years ( 25 to 75 ; standard deviation (SD) 15). There were 10 men and 24 women. All the patients suffered from at least Grade 2 of DRUJ instability by the radioulnar stress test intraoperatively. According to AO/OTA classification, nine fractures were Type 2R3A2, eight fractures were Type 2R3A3, seven fractures were Type $2 R 3 C 1$, and ten fractures were Type $2 \mathrm{R} 3 \mathrm{C} 2$. The mean duration from injury to surgery was 5 days ( 0 to $11 ; \mathrm{SD} 3)$. The mean intraoperative fracture gap after radius distraction was $2.2 \mathrm{~mm}$ (1.7 to 3.1; SD 0.3). The mean total operative time was 54 minutes (48 to 65 ; SD 5). The mean follow-up period was 44 months ( 37 to 56 ; SD 5). The above patient demographic data was presented in Table 1. After the surgery, all 34 patients had uneventful distal radius union at mean union time of 12 weeks (8 to 20; SD 3), and there was weak relation without statistical significance between fracture gap after radius distraction and union time $(-0.11, p=0.52)$. Meanwhile, 18 of 24 ulnar styloid fractures achieved union at final follow-up. For ulnar variance, it increased from $-1.4 \mathrm{~mm}(-2.4$ to 1.2 ; SD 0.8$)$ at post-operative 1 month follow-up to $-1.2 \mathrm{~mm}$ $(-2.2$ to $2.2 ;$ SD 1.1$)$ at the final follow-up with statistical difference $(p<0.05)$, and it also showed statistical difference $(p<0.05)$ when comparing injured wrist and contralateral uninjured wrist (mean 0.2 $\mathrm{mm} ;-0.5$ to $2.0 ;$ SD 0.6 ) at the final follow-up. These radiographic results were presented in Table 2.

Figure 2 demonstrated one of our case series receiving radius distraction from pre-operative status to the final follow-up status. 
Table 2

Radiographic, patient-rated and functional outcomes after radius distraction

\section{Radiographic Outcomes}

Union time, weeks (range, SD)

Ulnar styloid nonunion, number

Ulnar variance, $\mathrm{mm}$ (range, SD)

Post-operative 1-month, injured wrist

Post-operative final (minimal 36 months), injured wrist

Post-operative final (minimal 36 months), contralateral uninjured wrist

\section{Patient-rated and Functional Outcomes}

NRS of pain, number (range; SD)

DASH, score (range; SD)

MMWS, score (range; SD)

DRUJ instability

Grade 0, injured wrist, number

Grade 1, injured wrist, number

Grade 0 , contralateral uninjured wrist, number

Grade 1, contralateral uninjured wrist, number

Flexion-Extension, degree (range; SD)

Flexion

Extension

Pronation-Supination, degree (range; SD)

79.4 (60 to $90 ; 10.4)$

Pronation

$83.0(65$ to $90 ; 7.9)$

Supination

Radio-ulnar deviation, degree (range; SD)

24.9 (12 to $32 ; 7.2)$

Radial deviation

38.5 (25 to $50 ; 6.2)$

Ulnar deviation

SD: standard deviation; NRS: numeric rating scale; DASH: The Disabilities of the Arm, Shoulder questionnaire, and Hand; MMWS: Mayo modified wrist score

\section{Patient-rated outcome}


The mean NRS of pain of the injured wrists was 1 ( 0 to 2 ; SD 1) at the final follow-up. The mean MMWS scores and DASH scores of the injured wrists were 87 (60 to 100; SD 10) and 9 ( 0 to 21 ; SD 6$)$ at the final follow-up.

\section{Functional outcome}

For DRUJ instability outcome of the injured wrists, 32 patients had Grade 0 of DRUJ instability, and 2 patients had Grade 1 of DRUJ instability; on the other hand, 26 patients had Grade 0 of DRUJ instability, and 8 patients had Grade 1 of DRUJ instability of contralateral uninjured wrists. For ROM outcome of the injured wrists, the mean ROM of the injured wrists were $70.5^{\circ}$ in flexion (45 to 90 ; SD $11.2 ; 86 \%$ of contralateral side), $71.5^{\circ}$ in extension (50 to 90 ; SD $8.7 ; 90 \%$ of contralateral side), $26.1^{\circ}$ in radial deviation ( 12 to 35 ; SD $6.5 ; 81 \%$ of contralateral side), $36.2^{\circ}$ in ulnar deviation ( 25 to $45^{\circ}$; SD $4.3^{\circ} ; 92 \%$ of contralateral side), $79.3^{\circ}$ in pronation (60 to $90 ;$ SD $10.0 ; 96 \%$ of contralateral side), and $83.2^{\circ}$ in supination ( 65 to 90 ; SD $7.9 ; 99 \%$ of contralateral side).

\section{Complications}

No major complications were noted in any patient. One patient had a suture abscess that was treated successfully with suture removal, oral antibiotics, and debridement in operation room without admission. Two of our case series complained of persistent ulnar wrist pain for 1 year and one of our case series had painful scar within post-operative 6 months, and these symptoms were recovered gradually by medical intervention and physical therapy. There were no tendon complications, carpal tunnel syndrome or complex regional pain syndrome during the study period.

\section{Discussion}

While successful surgical treatments to confer DRUJ stability were well-documented, most of these treatments comprised either prolong immobilization or intrinsic TFCC repair or extrinsic ligament reconstruction following internal fixation of DRF (13-19), which may result in joint stiffness and delayed functional recovery for prolong immobilization, and the steep learning curve of repair or reconstruction of soft-tissue stabilizers for the DRUJ may hinder the application of these treatments to the setting of acute injury.

From biomechanical perspective of DRUJ stability, TFCC served as the primary static stabilizer while DIOM, though with some debate in recent biomechanical study (20), served as the secondary static stabilizer, and Arimitsu et al had been proved that the osteotomy performed proximal to the ulnar origin of the DIOM during ulnar shortening has better DRUJ stability compared to the distal osteotomy, especially in the coexistence of the DOB (8). The origin of the DOB was located at the distal ulnar proximal to the ulnar head while the insertion of the DOB was located at the sigmoid notch of the radius (21). Therefore, it is possible to tighten the DOB by moving the distal fracture fragment of DRF containing the insertion of the DOB distally, thereby resembling the biomechanical effect of the DOB in stabilizing DRUJ during proximal ulnar shortening. On the other hand, previous anatomy and biomechanics review had suggested that DRUJ instability in Galeazzi type fracture could be managed by anatomical reduction of radius 
fracture due to regain of the DOB's tension (22). Hence, we hypothesized that radius distraction could treat intraoperative DRUJ instability during volar plating of DRF by further DOB tightening, and our previous preliminary study (9) demonstrated the stabilizing effect of radius distraction to treat intraoperative DRUJ instability following volar plating fixation of acute DRF. However, it was unknown whether radius distraction could ameliorate this DRUJ instability in long-term follow-up. Therefore, we conduct this retrospective study to check long-term outcome of radius distraction on stabilizing DRUJ, and the results of our case series showed that radius distraction could not only prevent DRUJ from symptomatic instability necessitating revision surgery in long term follow-up, but also provide compatible functional outcome compared with other surgical technique dealing with DRF-related DRUJ instability $(13,15)$.

For ulnar variance measurement, we measured the ulnar variance at post-operative 1 month rather than post-operative immediately to ensure that the radiograph of injured wrist was taken in the same neutral position to avoid change of ulnar variance by different pronation-supination (23). There were 30 patients with negative ulnar variance in our case series at the final follow-up, and none of them suffered from avascular necrosis of lunate bone or other radiocarpal arthritis; this result also corresponds to the finding of the recent biomechanical study that radius distraction beyond native ulnar variance may not cause excessive loading of the radial-lunate contact stress (24), which may affect the metaphyseal bony purchase of volar locking plate, leading to distal screw migration and shortening of radial length as well as increase of ulnar variance. Although there was a statistical difference comparing the ulnar variance at post-operative 1-month follow-up and at the final follow-up, the significant statistical difference of ulnar variance between the injured wrists and the uninjured wrists at the final follow-up had proved the promising longevity of radius distraction. On the other hand, the distraction distance judging from ulnar variance seems to be too small to make the stabilizing effect feasible. However, the biomechanics study by Arimitsu et al(8) had found that significantly stability of the DRUJ could obtained with even $1 \mathrm{~mm}$ of proximal ulnar shortening, and the anatomy study by Moritomo et al(25) had found that the DOB acts as isometric stabilizer and its average length was about $25.4 \mathrm{~mm}$ to $26 \mathrm{~mm}$, and typical load-deformation curve from tendons and ligaments had revealed that tendons and ligaments can be strained to between 5 and $7 \%$ without damage(26). Therefore, we could find that the mean distracted length of the DOB without damage should be $1.3 \mathrm{~mm} \sim 1.8 \mathrm{~mm}$, which corresponded with the results of radius distraction in terms of the difference of ulnar variance, mean $1.4 \mathrm{~mm}$ at the final follow-up, from both hands in our case series.

For long-term DRUJ stability, all the patients got firm endpoint from the radioulnar stress test at the final follow-up. Although none of the patients suffered from symptomatic DRUJ translation or subluxation from the radioulnar stress test, there were still two cases with Grade 1 of DRUJ instability. Both patients with Grade 1 of DRUJ instability had increase of ulnar variance $(2.2 \mathrm{~mm}$ and $1.2 \mathrm{~mm}$, in each) at the final follow-up compared to the ulnar variance $(0.5 \mathrm{~mm}$ and $0 \mathrm{~mm}$, in each) at 1 month after the surgery. We assumed that the loss of ligamentotaxis by fracture subsidence could cause loosening of the DOB, which may lead to instability of DRUJ if initial DRUJ stabilizers other than the DOB were not fully healed. Meanwhile, since the prevalence of TFCC injuries increases with age (27), it is possible that their injured 
wrist might have suffered from inherent degenerative TFCC injuries as their contralateral uninjured wrist, which was not uncommon in our case series.

The role of fixation for ulnar styloid fracture in volar plating of DRFs is still a controversial issue. Traditionally, ulnar styloid fractures with concomitant DRFs were treated conservatively (28). There have been some studies suggesting that the outcome of DRFs was not significantly affected by fixation of ulnar styloid fracture (29-35). Fixation of ulnar styloid fracture after distal radius volar plating is generally not suggested if no DRUJ instability is detected $(29,36)$. Therefore, none of our cases with ulnar styloid fracture got internal fixation since intraoperative DRUJ stability was successfully achieved after radius distraction. Moreover, these 24 cases had Grade 0 of DRUJ instability at the final follow-up, and there was no significant difference in NRS of pain score, DASH, or MMWS between the cases of ulnar styloid nonunion and other cases with ulnar styloid fracture in our series at the final follow-up. Although ulnar styloid fracture could lead to DRUJ instability and hinder the outcome of DRFs with concomitant ulnar styloid fracture in some literature review $(2,37)$, it seems that the stabilizing effect of DIOM tightening in radius distraction could confer long term DRUJ stability even in the presence of ulnar styloid fracture, thereby achieving satisfactory functional outcomes no matter ulnar styloid is healed or not.

There are several limitations to mention in our study. First, our study was retrospective and the final case volume for cohort study was relatively small due to long-term follow-up. Second, we do not have a control group without radius distraction for comparison since it always needs surgical intervention for persisted DRUJ instability after fixation of DRFs to avoid prolong immobilization, and therefore we compared our results with the results in existing literature about DRF-related DRUJ instability. Third, there was no direct radiographic evidence of radius distraction due to lack of radiographic image prior to injury as comparator, and some radiographic parameters such as radial inclination, volar tilt, radial shift, and are not recorded, which may affect functional scores of the wrist joint and the relative stability of the DRUJ. Forth, the radioulnar stress test is not objective; since the radioulnar stress test has been proved to possess high inter-rater agreement (38) and we added two blinded evaluators to determine the DRUJ stability during the clinic visit. Although we didn't perform reliability test in this study, we considered it as a practical examination to detect DRUJ instability following the fixation of DRF. Fifth, we didn't explore the pathogenesis of DRUJ instability such as TFCC injury and DRUJ ligament injury by either arthroscopy or magnetic resonance imaging. Sixth, there were only plain film radiograph for pre/post-operative evaluation, and it would be better to evaluate the presence of osteoarthritis of DRUJ or degree of DRUJ subluxation from computed tomography scan or magnetic resonance imaging. Lastly, there was no direct biomechanical evidence to prove that tightened DOB alone in DRFs with intraoperative DRUJ instability could help the DRUJ to regain stability, and further biomechanical study focusing on the DOB's role in DRUJ stability should be considered to make this inference more persuasive.

\section{Conclusion}

In conclusion, our study indicated that radius distraction to treat intraoperative DRUJ instability during volar plating fixation of DRFs could maintain long-term stable DRUJs and satisfactory functional 
outcome, even in the presence of ulnar styloid fracture.

\section{Declarations}

- Ethics approval and consent to participate

This study has been performed in accordance with the ethical standards in the 1964 Declaration of Helsinki and has been carried out in accordance with relevant regulations of the US Health Insurance Portability and Accountability Act (HIPAA). The study was approved as simple retrospective study by the Institutional Review Board of Taipei Veterans General Hospital, Taiwan (2021-02-005AC).

- Consent for publication

Not applicable.

\section{- Availability of data and materials}

The datasets used and analyzed during the current study are available from the corresponding author on reasonable request under the regulation of the Institutional Review Board of Taipei Veterans General Hospital, Taiwan.

\section{- Competing interests}

The authors declare no potential conflicts of interest with respect to the research, authorship, and/or publication of this article.

\section{- Funding}

The authors received no financial support for the research, authorship, and/or publication of this article.

\section{- Authors' contributions}

Cheng-Yu Yin was involved in data analysis, writing the first draft of the manuscript and functional evaluation during post-operative follow-up. Hui-Kuang Huang was involve in revising manuscript and functional evaluation during post-operative follow-up. Duretti Fufa was involved in protocol development and English writing. Jung-Pan Wang was involved in gaining ethical approval, patient dataset recruitment and radiographic/functional evaluation during the whole study period.

\section{- Acknowledgements}

Not applicable.

\section{References}


1. Zimmerman RM, Jupiter JB. Instability of the distal radioulnar joint. J Hand Surg Eur Vol. 2014;39(7):727-38.

2. Sammer DM, Chung KC. Management of the distal radioulnar joint and ulnar styloid fracture. Hand Clin. 2012;28(2):199-206.

3. Kwon BC, Seo BK, Im HJ, Baek GH. Clinical and radiographic factors associated with distal radioulnar joint instability in distal radius fractures. Clin Orthop Relat Res. 2012;470(11):3171-9.

4. Kim JK, Yi JW, Jeon SH. The effect of acute distal radioulnar joint laxity on outcome after volar plate fixation of distal radius fractures. J Orthop Trauma. 2013;27(12):735-9.

5. Cheng HS, Hung LK, Ho PC, Wong J. An analysis of causes and treatment outcome of chronic wrist pain after distal radial fractures. Hand Surg. 2008;13(1):1-10.

6. Andersson JK AP, Strömberg J, Karlsson J, Fridén J. Patients with triangular fibrocartilage complex injuries and distal radioulnar joint instability have reduced rotational torque in the forearm. $\mathrm{J}$ Hand Surg Eur Vol. 2016;41(7):7.

7. Gofton WT, Gordon KD, Dunning CE, Johnson JA, King GJ. Soft-tissue stabilizers of the distal radioulnar joint: an in vitro kinematic study. J Hand Surg Am. 2004;29(3):423-31.

8. Arimitsu S, Moritomo H, Kitamura T, Berglund LJ, Zhao KD, An KN, et al. The stabilizing effect of the distal interosseous membrane on the distal radioulnar joint in an ulnar shortening procedure: a biomechanical study. J Bone Joint Surg Am. 2011;93(21):2022-30.

9. Wang JP, Huang HK, Fufa D. Radial Distraction to Stabilize Distal Radioulnar Joint in Distal Radius Fixation. J Hand Surg Am. 2018;43(5):493.e1-.e4.

10. Fujitani R, Omokawa S, Akahane M, lida A, Ono H, Tanaka Y. Predictors of distal radioulnar joint instability in distal radius fractures. J Hand Surg Am. 2011;36(12):1919-25.

11. Seo KN, Park MJ, Kang HJ. Anatomic reconstruction of the distal radioulnar ligament for posttraumatic distal radioulnar joint instability. Clin Orthop Surg. 2009;1(3):138-45.

12. Lindau T, Adlercreutz $C$, Aspenberg P. Peripheral tears of the triangular fibrocartilage complex cause distal radioulnar joint instability after distal radial fractures. J Hand Surg Am. 2000;25(3):464-8.

13. Gong HS, Cho HE, Kim J, Kim MB, Lee YH, Baek GH. Surgical treatment of acute distal radioulnar joint instability associated with distal radius fractures. Journal of Hand Surgery: European Volume. 2015;40(8):783-9.

14. Lee SK, Kim KJ, Cha YH, Choy WS. Conservative Treatment Is Sufficient for Acute Distal Radioulnar Joint Instability With Distal Radius Fracture. Ann Plast Surg. 2016;77(3):297-304.

15. Johandi F, Sechachalam S. Clinical and functional outcome of open primary repair of triangular fibrocartilage complex tears associated with distal radius fractures. J Orthop Surg (Hong Kong). 2017;25(1):2309499017690984.

16. Filius A, Zuidam JM, Jaquet JB, Slijper HP, Coert JH. Modification of the use of the extensor retinaculum for reducible distal radioulnar joint instability: technique and results. J Hand Surg Eur Vol. 2017;42(8):839-45. 
17. Aita MA, Mallozi RC, Ozaki W, Ikeuti DH, Consoni DAP, Ruggiero GM. Ligamentous reconstruction of the interosseous membrane of the forearm in the treatment of instability of the distal radioulnar joint. Rev Bras Ortop. 2018;53(2):184-91.

18. Carratala Baixauli V, Lucas Garcia FJ, Martinez Andrade C, Carratala Baixauli R, Guisasola Lerma E, Corella Montoya F. All-Arthroscopic Triangular Fibrocartilage Complex Ligamentoplasty for Chronic DRUJ Instability. Tech Hand Up Extrem Surg. 2019;23(1):44-51.

19. Lee SK, Chun YS, Bae JH, Yu YT, Choy WS. Arthroscopic Suture Repair With Additional Pronator Quadratus Advancement for the Treatment of Acute Triangular Fibrocartilage Complex Tear With Distal Radioulnar Joint Instability. Ann Plast Surg. 2019;83(4):411-8.

20. Trehan SK, Gould HP, Meyers KN, Wolfe SW. The Effect of Distal Radius Fracture Location on Distal Radioulnar Joint Stability: A Cadaveric Study. J Hand Surg Am. 2019;44(6):473-9.

21. Noda K, Goto A, Murase T, Sugamoto K, Yoshikawa H, Moritomo H. Interosseous membrane of the forearm: an anatomical study of ligament attachment locations. J Hand Surg Am. 2009;34(3):41522.

22. Moritomo $\mathrm{H}$. The distal interosseous membrane: current concepts in wrist anatomy and biomechanics. J Hand Surg Am. 2012;37(7):1501-7.

23. Jung JM, Baek GH, Kim JH, Lee YH, Chung MS. Changes in ulnar variance in relation to forearm rotation and grip. J Bone Joint Surg Br. 2001;83(7):1029-33.

24. Isa AD, McGregor ME, Padmore CE, Langohr DG, Johnson JA, King GJW, et al. Effect of Radial Lengthening on Distal Forearm Loading Following Simulated In Vitro Radial Shortening During Simulated Dynamic Wrist Motion. J Hand Surg Am. 2019;44(7):556-63 e5.

25. Moritomo H, Noda K, Goto A, Murase T, Yoshikawa H, Sugamoto K. Interosseous membrane of the forearm: length change of ligaments during forearm rotation. J Hand Surg Am. 2009;34(4):685-91.

26. Mow VC, Huiskes R. Basic Orthopaedic Biomechanics and Mechano-Biology. Philadelphia, UNITED STATES: Wolters Kluwer Health; 2004.

27. Chan JJ, Teunis T, Ring D. Prevalence of triangular fibrocartilage complex abnormalities regardless of symptoms rise with age: systematic review and pooled analysis. Clin Orthop Relat Res. 2014;472(12):3987-94.

28. Yuan C, Zhang H, Liu H, Gu J. Does concomitant ulnar styloid fracture and distal radius fracture portend poorer outcomes? A meta-analysis of comparative studies. Injury. 2017;48(11):2575-81.

29. Sammer DM, Shah HM, Shauver MJ, Chung KC. The effect of ulnar styloid fractures on patient-rated outcomes after volar locking plating of distal radius fractures. J Hand Surg Am. 2009;34(9):1595602.

30. Daneshvar P, Chan R, MacDermid J, Grewal R. The effects of ulnar styloid fractures on patients sustaining distal radius fractures. J Hand Surg Am. 2014;39(10):1915-20.

31. Nishiwaki M, Nakamura T, Nakao Y, Nagura T, Toyama Y. Ulnar shortening effect on distal radioulnar joint stability: a biomechanical study. J Hand Surg Am. 2005;30(4):719-26. 
32. Kim JK, Yun YH, Kim DJ, Yun GU. Comparison of united and nonunited fractures of the ulnar styloid following volar-plate fixation of distal radius fractures. Injury. 2011;42(4):371-5.

33. Tatebe M, Nishizuka T, Hirata H, Nakamura R. Ulnar shortening osteotomy for ulnar-sided wrist pain. J Wrist Surg. 2014;3(2):77-84.

34. Wijffels M, Keizer J, Buijze G, Zenke Y, Krijnen P, Schep N, et al. Ulnar styloid process nonunion and outcome in patients with a distal radius fracture: a meta-analysis of comparative clinical trials. Injury. 2014;45(12):1889-95.

35. Casadei K, Kiel J. Triangular Fibrocartilage Complex (TFCC) Injuries. StatPearls [Internet]: StatPearls Publishing; 2019.

36. Zenke Y, Sakai A, Oshige T, Moritani S, Nakamura T. Treatment with or without internal fixation for ulnar styloid base fractures accompanied by distal radius fractures fixed with volar locking plate. Hand Surg. 2012;17(2):181-90.

37. May MM, Lawton JN, Blazar PE. Ulnar styloid fractures associated with distal radius fractures: incidence and implications for distal radioulnar joint instability. J Hand Surg Am. 2002;27(6):96571.

38. Scheer JH, Hammerby S Fau - Adolfsson LE, Adolfsson LE. Radioulnar ratio in detection of distal radioulnar joint instability associated with acute distal radius fractures. J Hand Surg Eur Vol. 2010;35(9):730-4.

\section{Figures}
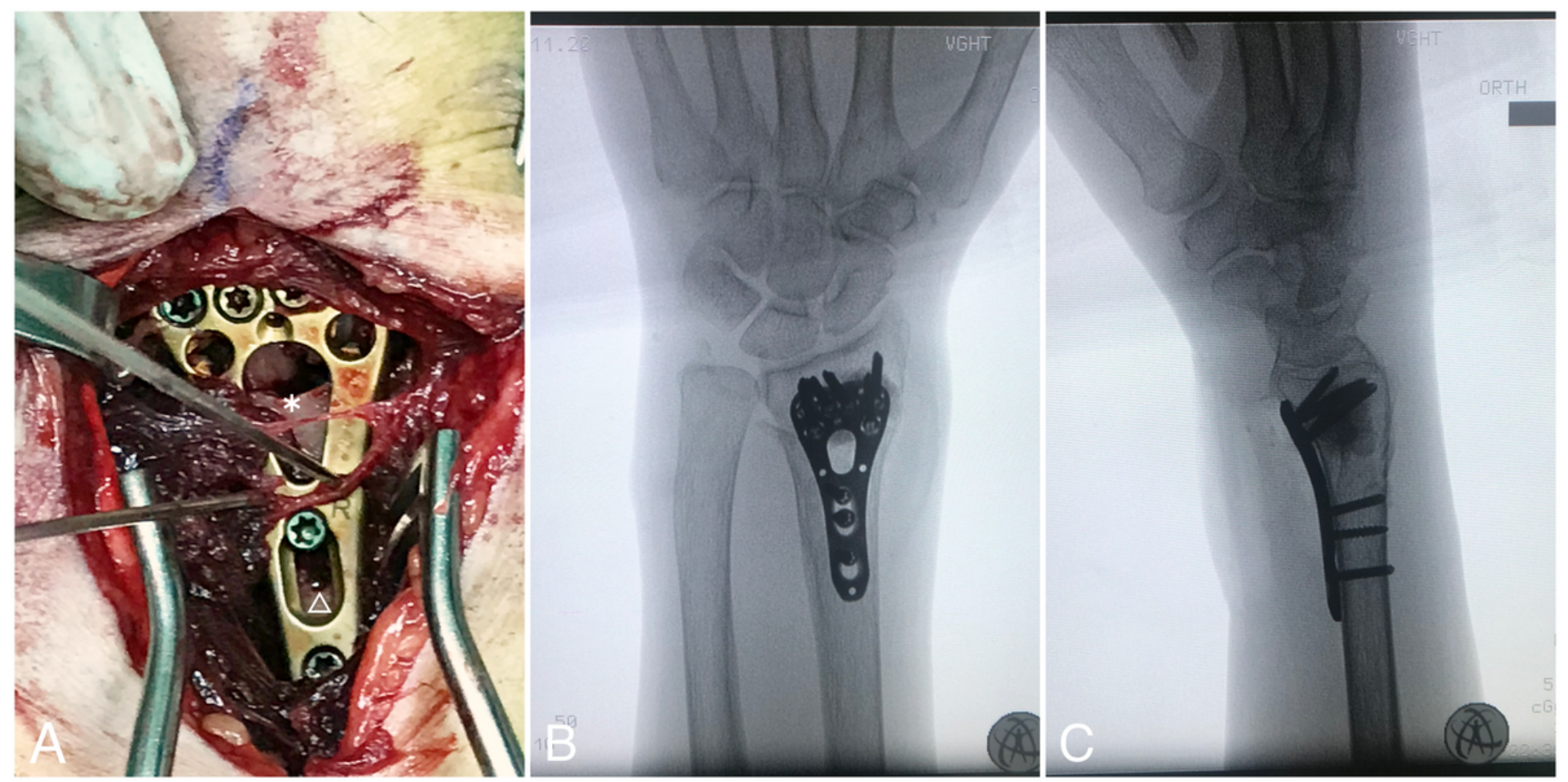

Figure 1 
Intraoperative demonstration of radius distraction (A) Intraoperative photo revealing the gap formation of radius distraction (asterisk) and oblong hole pending compression screw refastening (triangle) (B) anteroposterior view of radius distraction with bony defect filled with artificial bone graft (C) lateral view of radius distraction with bony defect filled with artificial bone graft

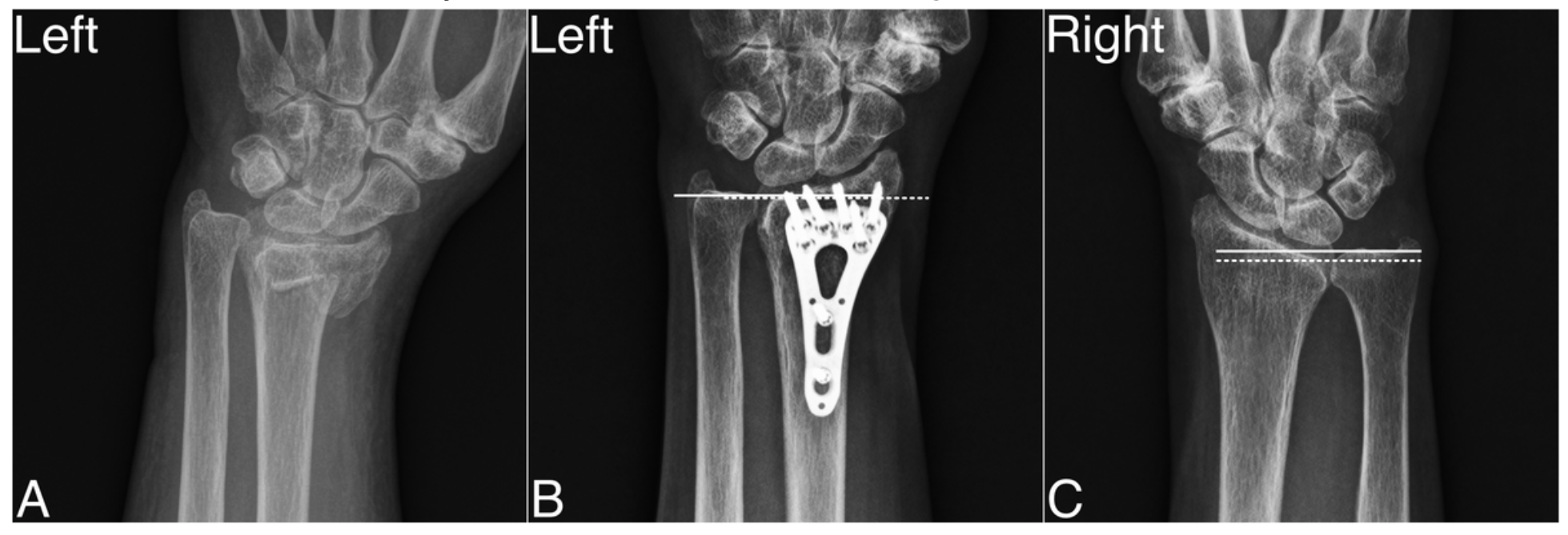

Figure 2

Long-term demonstration of the case receiving radius distraction (A) Pre-operative status of the injured wrist (B) The final status of the injured wrist with neutral ulnar variance (C) The contralateral uninjured wrist at the final follow-up with positive ulnar variance. (Dotted white line: distal articular surface of radius bone; white line: distal articular surface of ulnar bone)

\section{Supplementary Files}

This is a list of supplementary files associated with this preprint. Click to download.

- DRUJstresstestdemo.mp4 